\title{
A Modularity Bug in Java 8
}

\author{
Simon KRAMER \\ simon.kramer@a3.epfl.ch
}

December 31, 2016

\begin{abstract}
We demonstrate a modularity bug in the interface system of Java 8 on the practical example of a textbook design of a modular interface for vector spaces. Our example originates in our teaching of modular objectoriented design in Java 8 to undergraduate students, simply following standard programming practices and mathematical definitions. The bug shows up as a compilation error and should be fixed with a language extension due to the importance of best practices (design fidelity).
\end{abstract}

Keywords: component-based software construction, interface specifications, object-oriented programming, programming-language pragmatics

\section{Introduction}

We demonstrate a modularity bug in the interface system of Java 8 [1] on the practical example of a textbook design of a modular interface for vector spaces, which are very important for many computer applications of linear algebra. Our design is textbook in the sense that we simply follow standard programming practices and mathematical definitions, as any mathematically-inclined teacher of object-oriented programming in Java would do. That is, we construct our design from its standard algebraic components, thus ending up with not only $a$ but actually the natural modular design (by the algebraic definition of vector spaces), which however - and to our great surprise and embarrassment to our students - fails compilation due to an important expressiveness limitation of the Java 8 language. This limitation resides in its (not so) generic type system and should be remedied with an appropriate language extension, for the sake of keeping best practices best and saving our faces to our students of Java.

Nota bene We do not claim that it be impossible to model vector spaces in Java 8; it is possible. What we do claim is that it is impossible to specify with interfaces and implement with corresponding classes such spaces in Java 8 in the natural modular way, whereby we mean reflecting their algebraic structure with its definitional components (design fidelity). So the great modularity promise of component-based software construction with object-oriented programming in 
Java has not been fulfilled yet, even after more than 20 years. In particular (counterexample), it cannot be fulfilled in Java 8 when combining such basic components as a field of scalars (e.g., rational numbers) and an additive group of vectors of such scalars so as to form a vector space (e.g., over rationals). Unfortunately, not even the planned Java 9 module system [2]

- representing "a fundamental shift to modularity as a first-class citizen for the whole Java platform" and

- in which "interfaces play a central role"

offers the required basic modularity, perhaps because of prioritising packages (coarser-grained modularity through import and export control) over interfaces (finer-grained modularity through multiple-inheritance-based composition).

\section{The bug}

We demonstrate the modularity bug in the interface system of Java 8 by constructing our interface (specification) for vector spaces bottom-up from its standard algebraic (interface) components, combining them through multiple inheritance when needed. Luckily, Java 8 offers multiple inheritance from interfaces. Recall that from classes, Java 8 only offers single inheritance. Our following interface components specify the algebraic operations up to our sought vector spaces, however without stipulating their corresponding algebraic (equational) laws (constraints). It would be nice if also this could become possible in a future release of Java in which interfaces could carry such algebraic structure, $\grave{a} l a$ algebraic specifications [3]. In particular, the commutativity of commutative rings is left implicit (unspecified) in the following corresponding interface (Page 4 ).

Our interfaces are presented as divided up into non-problematic and problematic components. The non-problematic interface components are meant to illustrate important modular (algebraic) interface specifications that are possible in Java 8 (though unfortunately still without algebraic constraints). The problematic interface components are meant to demonstrate an important modular (algebraic) interface specification that is not possible in Java 8, namely the one for vector spaces (our bug). The frontier between the non-problematic and

the problematic is our empirical evidence for our claimed expressiveness limitation in Java 8 and motivates our suggested language extension therefor.

\section{$2.1 \quad$ Non-problematic interfaces}

Our first (atomic) interface component for vector spaces is the following one for (commutative) additive semigroups. These carry one binary operation, here called plus, specified with an explicit parameter right, with the corresponding parameter left left implicit, as is typical in object-oriented programming languages. (We can refer to this implicit parameter with the Java-keyword this.) 
algebra.AdditiveSemigroup

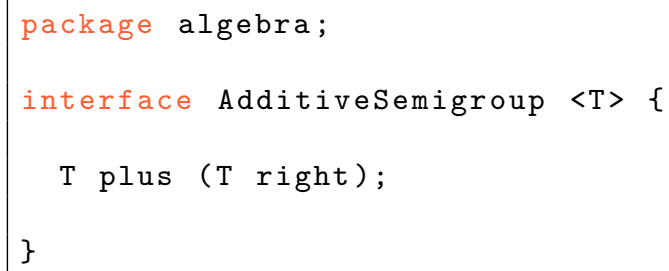

A typical example of this strucure are the natural numbers (naturals) with addition. They actually form also a (commutative) multiplicative semigroup (and more), which is our second atomic interface component for vector spaces.

algebra.MultiplicativeSemigroup

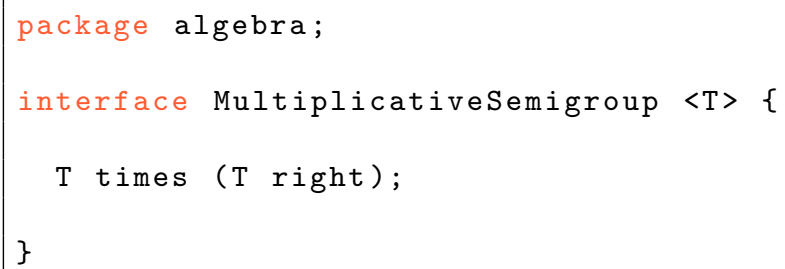

Our next (compound) interface component for vector spaces is the following one for additive monoids, which we obtain through single inheritance from our interface component for additive semigroups and through the addendum of a getter method for the additionally required additive neutral element.

algebra.AdditiveMonoid

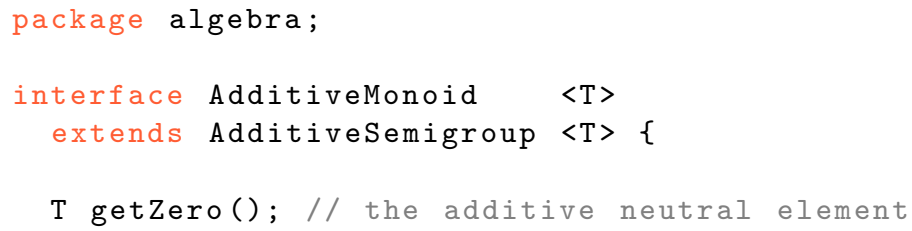

As a typical example, the naturals form (also) an additive monoid. Moreover, they form a multiplicative monoid, which is similar to its additive counterpart.

algebra.MultiplicativeMonoid

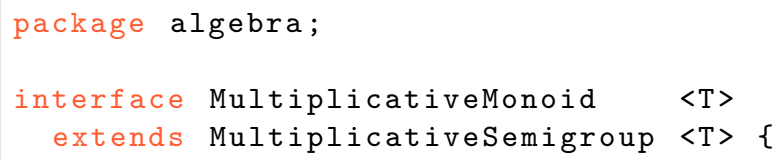




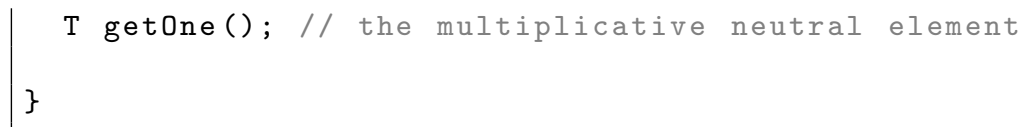

Our next, further compound interface component for vector spaces is the following one for additive groups, which we obtain through single inheritance from our interface component for additive monoids and through the addendum of a getter method for the additionally required additive inverse element.

\section{algebra.AdditiveGroup}

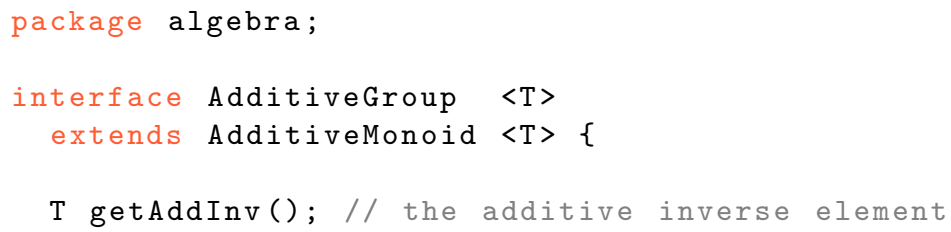

As a typical example, the integer numbers (integers) form an additive group. Moreover, they form a commutative ring, which we obtain through multiple (double) inheritance from our (two) interface components for additive groups on the one hand and for multiplicative monoids on the other hand.

algebra.CommutativeRing

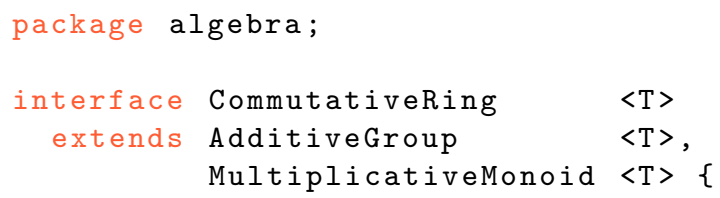

Our next compound interface component for vector spaces is the following one for multiplicative groups, which we obtain through single inheritance from our interface component for multiplicative monoids and through the addendum of a getter method for the additionally required multiplicative inverse element.

algebra.MultiplicativeGroup

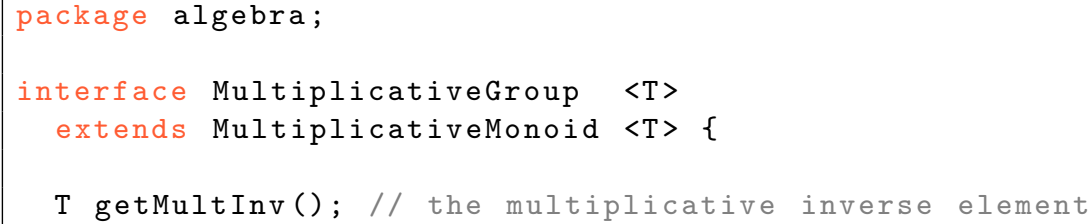


Our final non-problematic, further compound interface component for vector spaces is the following one for fields, which we obtain through

- multiple (double) inheritance from our (two) interface components for additive groups on the one hand and for multiplicative groups on the other hand, and

- the overriding of the getter method for the multiplicative inverse element from the inherited multiplicative group with an exception for division by zero. (Of course, we could define a more general, algebraic exception instead of getting by with the predefined arithmetic exception.) Luckily, such an exceptional overriding is possible with Java 8 multiple inheritance.

algebra.Field

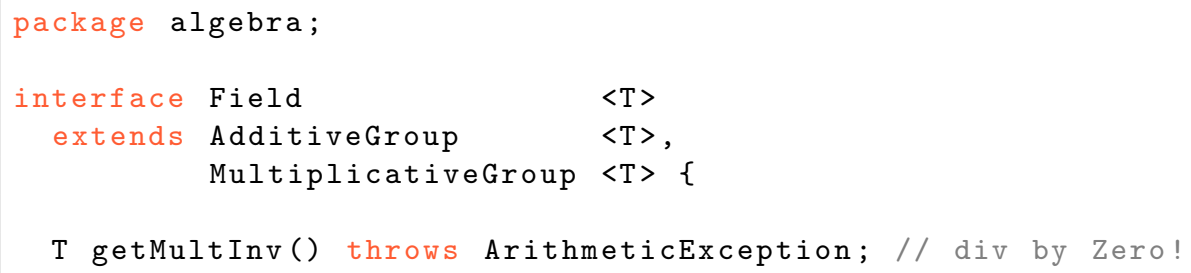

A typical example of a field are the rational numbers (rationals) with addition and multiplication, of course including subtraction and division through the inherited additive and the inherited multiplicative inverse elements, respectively.

\subsection{Problematic interfaces}

The desideratum of an interface for vector spaces in Java is the one displayed in the upper part of Table 1 . It is simply the one that the mathematical (algebraic) definition requires. We obtain it through multiple (double) inheritance from our (two) interface components for an additive group of vectors on the one hand and for a field of scalars on the other hand, and through the addendum of a method for the scalar multiplication of vectors. Very unfortunately, the desired interface produces the compilation errors displayed in the lower part of Table 1 . which cannot be remedied in Java 8 due to the limited expressiveness of its (not so) generic type system. Even the simpler, less desirable, because type-wise less precise interface for vector spaces displayed in the upper part of Table 2 produces an intrinsic compilation error, which is displayed in the lower part. (An even simpler interface would be a simplistic specification for vector spaces.)

Hence, multiple inheritance from interfaces with different generic types is problematic in Java 8. This state of affairs has preempted - and still preempts, even in the future Java 9 [2] - the modularity of many interface specifications and thus has worsened best practices in Java for more than 20 years. 
